\title{
Korrespondierende Autorin:
}

Prof. Dr. Anita Riecher-Rössler

Universitätsspital Basel

Petersgraben 4

CH-4031 Basel

E-Mail: RiecherA@uhbs.ch

https://webmail.unibas.ch/imp/message.php?index=1689 - \#

Tel.-Nr. 0041612655114

Fax 0041612654599

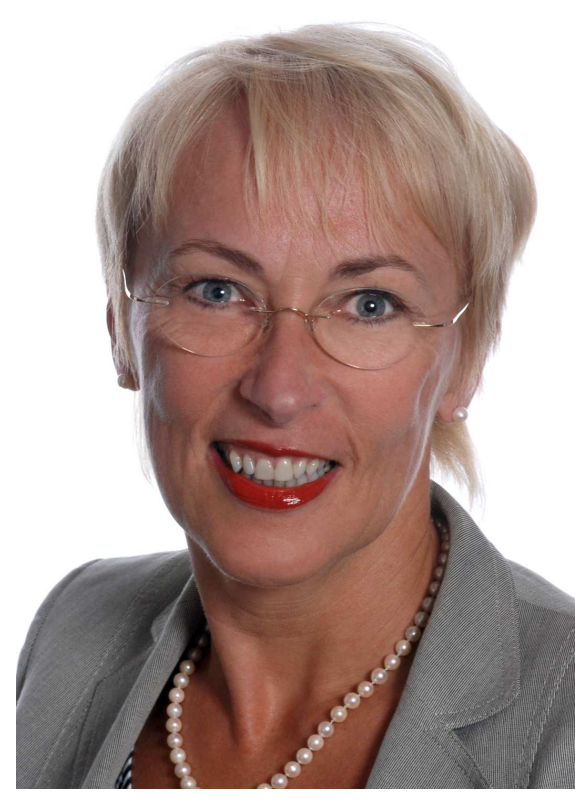

Interessenkonflikt: Es besteht kein Interessenkonflikt. 


\title{
Schwangerschaftsdepression und deren Behandlung
}

\author{
A. Bader ${ }^{1,2}$, U. Frisch ${ }^{2}$, A. Wirz-Justice ${ }^{1}$, A. Riecher-Rössler ${ }^{2}$ \\ ${ }^{1}$ Zentrum für Chronobiologie, Universitäre Psychiatrische Kliniken Basel \\ ${ }^{2}$ Psychiatrische Poliklinik, Universitätsspital Basel
}

\section{Zusammenfassung}

Etwa $11 \%$ aller schwangeren Frauen leiden unter einer behandlungsbedürftigen Depression, welche unbehandelt mit Risiken wie Frühgeburt oder niederem Geburtsgewicht verbunden ist. Da manche Symptome der Depression oft der Schwangerschaft zugeschrieben werden, ist die Diagnose einer Schwangerschaftsdepression nicht immer leicht. Eine weitere Herausforderung ist die Wahl der geeigneten Therapiemaßnahme. Als Behandlungsmöglichkeiten stehen neben Psycho- und Pharmakotherapie auch Elektrokrampftherapie (EKT) sowie Lichttherapie zur Auswahl. Aktuelle Berichte über Auswirkungen von Antidepressiva auf die Entwicklung des Ungeborenen und postpartale Anpassungsschwierigkeiten führen zu großen Verunsicherungen. Die Entscheidung, welche Therapiemaßnahme ergriffen wird, kann nur mit der werdenden Mutter zusammen getroffen werden; ein sorgfältiges Abwägen der Vor- und Nachteile der Behandlung ist dafür Voraussetzung.

\section{Schlüsselwörter:}

Schwangerschaftsdepression - Behandlung - Antidepressiva - Psychotherapie - Lichttherapie

\section{Depression during Pregnancy and its Treatment}

\section{Summary}

About $11 \%$ of pregnant women suffer from a major depression requiring treatment. If left untreated, there are risks such as preterm delivery or low birth weight. The initial difficulty lies in diagnosing the depression itself, since many depressive symptoms can be ascribed to the pregnancy. A further challenge is choosing the appropriate therapy. Treatment possibilities are psychotherapy, antidepressants, electroconvulsive therapy (ECT), or the new possibility of light therapy. A growing number of reports on the side effects of antidepressants in pregnancy have led to uncertainties as to how to proceed. Thus, choosing the most suitable treatment needs to be 
made together with the pregnant woman; a careful clarification of possible risks attendant on each treatment option is essential.

\section{Key words:}

Depression - Pregnancy - Psychotherapy - Antidepressants - Light therapy 
Jede zehnte Schwangere leidet an einer behandlungsbedürftigen Depression. Die Schwangerschaft stellt für den Therapeuten eine Herausforderung dar, da zum einen unbehandelte Depressionen negative Folgen für die Mutter und das Kind haben können und zum anderen die Behandlungsangebote durch die Schwangerschaft (SS) selbst begrenzt erscheinen. Ziel des vorliegenden Übersichtsartikels ist es, einen Überblick über das gegenwärtige Wissen und die Behandlung von unipolaren Schwangerschaftsdepression (SSD) zu geben. Dabei gehen wir nicht auf bipolare Störungen ein, sondern verweisen diesbezüglich auf Sasse et al. [24]. Die Literaturrecherche erfolgte mit Hilfe von PubMed über Querverweise und Handbücher.

\section{Häufigkeit, klinisches Bild und Diagnostik}

Etwas $10.7 \%$ aller schwangeren Frauen leiden an einer Depression, wie eine Metaanalyse über 21 Studien berichtet [4]. Zu berücksichtigen ist dabei aber, dass die diagnostische Einschätzung „Depression“ in vielen der 21 Studien nicht anhand DSM-VI oder ICD-10-Kriterien vorgenommen wurde, sondern anhand von Selbstbeurteilungsfragebögen. Berechnet man die Prävalenz pro Schwangerschaftstrimenon, liegen die Werte im zweiten und dritten Trimenon mit $12 \%$ fast doppelt so hoch wie im ersten [12]. Diese Prävalenzraten weichen nicht signifikant von der Prävalenz depressiver Störungen bei Frauen im gebärfähigen Alter ab [6].

Auch im klinischen Bild unterscheidet sich die SSD nicht grundsätzlich von Depressionen außerhalb der Fertilitätszeit. Die Symptome sind prinzipiell die gleichen [18], doch werden sie in der SS durch die somatischen Schwangerschaftsbeschwerden geprägt. Die Frauen berichten häufiger über Übelkeit, Bauchschmerzen, Kurzatmigkeit, gastrointestinalen Probleme und sexuelle Dysfunktionen [16].

SSD bleiben häufig unerkannt. Eine kürzlich veröffentlichte Analyse zeigte, daß nur ca. $20 \%$ der schwangeren Frauen mit einer Depression entsprechend diagnostiziert wurden [14]. Zum einen verschweigen die betroffenen Frauen aus Schuld- und Schamgefühlen ihre Beschwerden, zum anderen sind die pathologischen Symptome der Depression schwer von den normalen körperlichen Veränderungen während einer SS zu unterscheiden. Eine standardisierte Erfassung von depressiven Symptomen während der SS wird darum empfohlen. 
In vielen Praxen und Frauenkliniken fehlten bisher praktikable Screeninginstrumente zur Erhebung von depressiven Symptomen bei schwangeren Frauen. Als zweckmäßiges Instrument hat sich die Edinburgh Postnatal Depression Scale (EPDS) erwiesen (Abbildung 1). Im Vergleich zu herkömmlichen Depressionsfragebögen verzichtet sie auf das Nachfragen von körperlichen Beschwerden und hat sich für einen Einsatz in der SS bewährt [20].

\section{Ursachen}

Als biologische Risikofaktoren für das Auftreten einer SSD gelten eine frühere Depression - ca. $43 \%$ der Frauen mit einer Depression in der Vorgeschichte erleben in der SS einen Rückfall [7] - sowie psychiatrische Erkrankungen in der Familienanamnese. Als psychosoziale Risikofaktoren werden unter anderem ein niedriger sozioökonomischer Status, eine ungenügende soziale Unterstützung, niedriges Einkommen, belastende Lebensereignisse, frühere Aborte, ambivalente Einstellung gegenüber der SS und Sorgen über die Gesundheit des Ungeborenen diskutiert.

\section{Risiken}

Die SSD konnte als Hauptrisikofaktor für die postpartale Depression identifiziert werden. Ungefähr ein Drittel der schwangeren Frauen, welche eine Depression erleben, erkranken später an ihr. Auch kann eine SSD in schweren Fällen zum Suizid führen, welcher die häufigste Todesursache in der SS ist [3].

Viele Studien berichten über einen Zusammenhang zwischen Depressivität, Frühgeburt und niedrigem Geburtsgewicht [13]. Hier scheint eine streßbedingte erhöhte Aktivität der mütterlichen Hypothalamus-Hypophysen-Nebennieren-Achse verantwortlich zu sein [1], [10]. Depressive Schwangere greifen häufiger nach Suchtmitteln und versäumen es häufiger, adäquate Geburtsvorbereitungen zu treffen [15]. Weiter werden ein erhöhtes Risiko für Präeklampsie, vorzeitige Wehen, unzulängliche Gewichtszunahme des Ungeborenen, operative Entbindungen, erhöhte fetale Herzrate als Reaktion auf mütterlichen Streß, Schlafstörungen beim Neugeborenen und negative Beeinflussung von Emotionsregulation und Temperament des Kindes diskutiert (Übersicht in [3]). 


\section{Behandlung der SSD}

Die Therapie der SSD stellt eine große Herausforderung dar, da insbesondere psychopharmakologische Strategien nicht ohne Risiko für das Ungeborene sind. Das therapeutische Ziel ist darum die psychische Stabilisierung der Mutter, ohne dem Ungeborenen dabei zu schaden. Auch bei der Schwangeren selbst besteht oft eine große Verunsicherung bezüglich der Medikation und deren negativen Auswirkungen. Um die Frauen in dieser Zeit optimal zu unterstützen und eine Verschlechterung der Symptomatik möglichst früh zu erkennen, sollte die Schwangere möglichst engmaschig betreut werden. Als Behandlungsoptionen stehen Psychotherapie (PT), Antidepressive Medikamente (AD), Elektrokrampftherapie (EKT) und ersten positiven Studien zufolge auch die Lichttherapie (LT), als chronobiologisches Verfahren, zur Verfügung.

Unabhängig von der gewählten Behandlungsmethode, ist eine vorausgehende sorgfältige Psychoedukation der Betroffenen über ihre Erkrankung notwendig. Hierdurch kommt es häufig schon zu einer gewissen Entlastung. Die Auswahl der Therapiemaßnahme sollte zusammen mit der Patientin erfolgen. Beim Abwägen der Vor- und Nachteile der Behandlungsoptionen empfiehlt es sich die NICE Guidelines (2007) zu beachten. Siehe hierzu Tabelle 1.

\section{Psychotherapie}

Die PT ist bei der Behandlung von SSD die Methode der ersten Wahl, insbesonders bei leichteren bis mittelgradigen Depressionen (Tabelle 2). Sie kann auch präventiv eingesetzt werden, wenn Frauen aufgrund der Planung einer SS ihr Medikament absetzen. Vorteile sind die fehlende Exposition des Ungeborenen mit psychotropen Substanzen und ein langanhaltender Therapie-Effekt, jedoch bei hohem zeitlichen und finanziellem Aufwand.

Neben einer supportiven Gesprächstherapie kommen als spezifische Psychotherapieformen die Interpersonelle Psychotherapie (IPT) und kognitive Verhaltenstherapie (KVT) infrage. Während verschiedene Studien die Wirksamkeit der KVT und IPT bei depressiven Erkrankungen nachweisen konnten, welche als Einzel- oder Gruppenbehandlung durchgeführt werden können, liegen über deren Einsatz in der SS nur wenige Wirksamkeitsstudien vor (Übersicht in [9]). Nur eine Studie erfüllt die Kriterien einer randomisierten Placebo-kontrollierten Studie [26], bei welcher eine für Schwangere modifizierte IPT, im Vergleich zu einem elterlichen Schulungsprogramm, zu signifikant besseren Ergebnissen führte. Dafür wurden die klassischen 
Themenbereiche der IPT (Trauer, interpersonelle Konflikte, Rollenübergänge, interpersonelle Defizite/Sensitivität) mit spezifischen „Problemen in der SS“ ergänzt.

Auch wenn gegen eine PT entschieden wird, bleibt eine therapeutische Intervention wünschenswert. Diese kann auch nur auf supportive Gespräche beschränkt sein, welche die Frauen von ihren Schuldgefühlen entlasten und eine Beratung über praktische Hilfen und Bewältigungsstrategien enthalten.

Weil bisher nur wenig über den Einsatz von PT bei SSD geforscht wurde, ist die Entwicklung weiterer Therapiemanuale und Durchführung von Wirksamkeitsstudien wünschenswert.

\section{Medikamentöse Behandlung}

Bei schweren Depressionen, oder wenn PT alleine nicht wirksam ist, ist immer auch zusätzlich eine Psychopharmakotherapie durchzuführen.

Psychopharmaka passieren die Plazenta und sind im Fruchtwasser nachweisbar. Der Embryo ist besonders in der Organogenese (ca. 15 bis 60 Tage nach der Befruchtung) gegenüber toxischen Einflüssen empfindlich, weshalb es in dieser Phase zu teratogenen Schäden kommen kann. In der anschließenden Fetalphase (2. und 3. Trimenon) nimmt diese Empfindlichkeit wieder ab [25]. Um die Geburt herum kann es zu perinatalen Komplikationen kommen, die meist vorübergehenden Naturen sind. Aussagen über Langzeitauswirkungen auf die Kindsentwicklung sind aufgrund der wenigen Langzeitstudien kaum möglich.

Jeder Medikation sollte ein gewissenhaftes Abwägen der Nutzen und Risiken für Mutter und Kind vorausgehen. Ausführliche Aufklärung der werdenden Mutter über mögliche Risiken und Nebenwirkungen müssen erfolgen.

Der Einsatz von mehreren Medikamenten zugleich soll vermieden werden. Monotherapie, evtl. in höherer Dosis, ist deshalb einer Kombination mehrerer Psychopharmaka vorzuziehen. Medikamentenwechsel erhöhen die Exposition des Ungeborenen. Vorzuziehen sind Medikamente mit geringem Metabolismus, einer höheren Eiweißbindungskapazität, welche den plazentaren Übertritt erschwert sowie solche mit einem geringen Interaktionspotential [8]. Tabelle 2 zeigt die Fälle, in welchen eine medikamentöse Therapie in der SS erfolgen sollte.

\section{Selektive-Serotonin-Reuptake-Hemmstoffe (SSRI)}


Teratogene Effekte von SSRIs sind bislang nicht eindeutig nachzuweisen, jedoch gibt es einige Publikationen, die auf ein leicht erhöhtes Risiko für verschiedene Fehlbildungen unter Paroxetin und eventuell auch unter Fluoxetin hinweisen [25]. Neue Studien fanden keinen Zusammenhang zwischen einer SSRI-Exposition im ersten Trimenon und fetalen Herzfehlbildungen [2, 17]. In jüngerer Zeit wurde jedoch ein signifikanter Zusammenhang zwischen SSRI-Exposition im letzten Trimenon und persistierendem pulmonalen Hypertonus des Neugeborenen beobachtet. Außerdem wurden vorübergehende Anpassungsstörungen nach der Geburt wie Zittrigkeit, Übererregbarkeit, mildere respiratorische Störungen wie vorübergehender Tachypnoe, schwaches Schreien, verminderter Tonus und vermehrte Intensiv-Überwachungsbedürftigkeit beschrieben (Übersicht in [25]).

Es gibt bisher nur einzelne Studien über die Langzeitentwicklung der Kinder nach SSRIExposition in der SS. Einige berichten über etwas häufiger aufgetretene psychomotorische Entwicklungsrückstände bei Kindern im Alter von 3 bis 40 Monaten, eine andere Studie konnte diesbezüglich keine bei 2 jährigen Kindern finden [25]. Insgesamt können aufgrund der Datenlage keine entwarnenden Aussagen über den Einsatz der SSRIs in der Schwangerschaft gemacht werden.

\section{Trizyklische Antidepressiva (TZA)}

Bei keinem der seit längerem gebräuchlichen Präparate konnte bisher der Verdacht auf teratogene Effekte bestätigt werden [25].

Bei einigen Neugeborenen wurden Entzugssymptome wie Zittrigkeit, Übererregbarkeit, Atemnotsyndrom und Krämpfe beobachtet, deren Müttern bis zur Geburt mit TZA behandelt wurden [25]. Neuere Studien konnten keinen signifikanten Zusammenhang zwischen pränataler TZA-Exposition und perinatalen Schwierigkeiten zeigen [23, 30].

Die Einnahme von TZA während der gesamten Schwangerschaft scheint keine arzneispezfischen Auffälligkeiten in der Entwicklung der Kinder zu provozieren [25]. TZA gelten deshalb als die am besten geeigneten AD in der SS.

\section{Monoaminooxidase-(MAO)-Hemmer}

Es besteht der Verdacht auf pränatal toxische Wirkungen dieser Wirkstoffe. Wachstumsretardierungen und fetale Toxizität sind berichtet worden. Insgesamt ist die Datenlage begrenzt, so daß zu einem Einsatz von MAO-Hemmern in der SS abzuraten ist [25]. 


\section{Andere Antidepressiva}

Die vorliegenden Daten für Duloxetin, Mirtazepin, Nefazodon, Venlafaxin und Bupropion sind begrenzt, es scheinen keine Hinweise auf Teratogenität zu bestehen [25]. Bezüglich der Risiken von Venlafaxin siehe Tabelle 3.

\section{Elektrokrampftherapie (EKT)}

Die EKT wird selten angewendet, auch wenn verschiedene Kasuistiken zeigen, daß die Anwendung in der SS relativ sicher und effektiv ist. Prospektive oder kontrollierte Studien gibt es nicht, die größte Metaanalyse über 300 Fälle stammt von Miller [19]. Die American Psychiatric Association empfiehlt die EKT bei schwer psychotischen oder akut suizidalen Frauen, wenn andere Therapiemaßnahmen versagt haben.

\section{Lichttherapie}

Die LT, eine chronobiologische, nicht-pharmakologische somatische Therapie könnte eine Alternative zu den AD sein. Die Chronobiologie geht davon aus, daß bei der Pathogenese von Depressionen Rhythmusstörungen der inneren Uhr und des Schlaf-Wachzykluses eine wichtige Rolle spielen - ob diese Ursachen oder Folgen der Depression sind, ist ungeklärt. Depressives Verhalten (Passivität, unstrukturierter Tagesablauf, wenige soziale Kontakte, verminderter Aufenthalt im Freien usw.) führt jedoch zu einer Verstärkung dieser Rhythmusstörungen [29]. Die chronobiologische Therapie der Depression versucht die veränderten endogenen circadianen Rhythmen mit dem äußeren Tag-Nacht-Zyklus zu resynchronisieren und dadurch eine Verbesserung der affektiven und physischen Symptome zu erreichen. Dies kann beispielsweise durch Lichtexposition mit therapeutischen Lampen von hinreichender Stärke und Dauer erfolgen. Die genaue physiologische antidepressive Wirkungsweise von Licht ist noch weitgehend unbekannt, doch bestehen neben der Wirkung auf die circadiane Rhythmik, weitere positive Einflüsse auf die Serotoninproduktion im ZNS.

Seit ca. 1980 wird Licht zur Behandlung von Winterdepressionen eingesetzt und führt bei 60\% bis $75 \%$ der Patienten zu einer signifikanten Verbesserung [27]. Auch bei nichtsaisonalen Depressionen ist LT mit einer Effektstärke von 0.55 (95\% CI=0.23-0.87) wirksam [28]. Vorteile sind die geringen Nebenwirkungen. Die Wirksamkeit bei SSD wurde bisher erst in zwei PilotStudien untersucht, weshalb noch keine klinischen Empfehlungen möglich sind. In der ersten 
Studie wurden 16 schwangere depressive Frauen täglich während drei Wochen mit Licht behandelt [22]. Wöchentliche Depressionsratings ergaben nach drei Wochen eine Verbesserung der Symptomatik von 49\%, nach fünf Wochen von 59\%, im Vergleich zum Ausgangswert. In einer zweiten randomisierten Placebo-kontrollierten Studie [11] führte die LT nach fünf Wochen zu einer Verbesserung der depressiven Symptomatik, welche nach 10 Wochen signifikant war. Um genaue Aussagen machen zu können, wird zur Zeit in Basel eine umfangreiche placebokontrollierete Studie an depressiven Schwangeren durchgeführt.

\section{Fazit für die Praxis}

Um depressive Erkrankungen bei schwangeren Frauen nicht zu übersehen, empfiehlt es sich, standardmäßig die EPDS als Screeninginstrument einzusetzen und bei Hinweisen auf eine SSD eine ausführliche Diagnostik durchzuführen. Der Entscheidungsprozeß über die Behandlungsmethode beinhaltet eine sorgfältige Aufklärung der werdenden Mutter und wenn möglich ihres Partners über mögliche Risiken unter Berücksichtigung des Schweregrades der Depression, des Risikos einer Medikamentenexposition des Ungeborenen und der negativen Auswirkungen einer unbehandelten Depression.

Obwohl dringend notwendig, gibt es noch nicht genügend effiziente und gleichwohl für die Gesundheit des Neugeborenen unbedenkliche Behandlungsmethoden. Die Entwicklung solcher ist von eminenter Bedeutung. Lichttherapie ist eine vielversprechende Alternative zu den Medikamenten, deren Wirksamkeit bei Schwangeren weiter überprüft werden muss. 


\section{Literatur}

1. Alder J, Fink N, Bitzer J et al. (2007) Depression and anxiety during pregnancy: A risk factor for obstetric, fetal and neonatal outcome? A critical review of the literature. J Maternal Fetal and Neonatal Med 20:189-209

2. Alwan S, Reefhuis J, Rasmussen SA et al. (2007) Use of selective serotonin-reuptake inhibitors in pregnancy and the risk of birth defects. NEJM 556:2684-2692

3. Bennett HA, Einarson A, Taddio A et al. (2004) Depression during pregnancy: overview of clinical factors. Clin Drug Investig 24:157-179

4. Bennett HA, Einarson A, Taddio A et al. (2004) Prevalence of depression during pregnancy: Systematic review. Am Coll Obstet Gyn 103:698-709

5. Bergant AM, Nguyen T, Heim K et al. (1998) German language version and validation of the edinburgh postnatal depression scale. Dtsch Med Wochenschr 123:35-40

6. Bitzer J, Riecher-Rössler A. (2005) Was sind Frauenleiden? Epidemiologische Aspekte. In: Riecher-Rössler A, Bitzer J (eds), Frauengesundheit. Ein Leitfaden für die ärztliche und psychotherapeutische Praxis. Elsevier GmbH, Urban und Fischer Verlag, München, $13-30$

7. Cohen LA, Altshuler LL, Harlow BL et al. (2006) Relapse of major depression during pregnancy in women who maintain or discontinue antidepressant treatment. JAMA 295:499-507

8. Dayan J, Creveuil C, Herlicoviez M et al. (2002) Role of anxiety and depression in the onset of spontaneous preterm labor. Am J Epidemiol 155:302-304

9. Dennis C-L, Ross Le, Crigoriadis S (2007) Psychosocial and psychological interventions for treating antenatal depression. Cochrane Database of Systematic Reviews, Issue 3. Art. No.: CD006309. DOI: 10.1002/14651858.CD006309.pub2:1-8

10. Ehlert U (2004) Einfluss von Stress auf den Schwangerschaftsverlauf und die Geburt. Psychotherapeut 49:367-376

11. Epperson CN, Terman M, Terman JS et al. (2004) Randomized clinical trial of bright light therapy for antepartum depression: Preliminary findings. J Clin Psychiatry 65:421425

12. Evans J, Heron J, Francomb H et al. (2001) Cohort study of depressed mood during pregnancy and after childbirth. BMJ 323:257-260

13. Evans J, Heron J, Patel RR et al. (2007) Depressive symptoms during pregnancy and low birth weight at term: longitudinal study. Br J Psychiatry 191:84-85

14. Hatton DC, Harrison-Hohner J, Matarazzo J et al. (2007) Missed antenatal depression among high risk women: a secondary analysis. Arch Womens Ment Health 10:121-123

15. Kelly HK, Russo J, Holt VL et al. (2002) Psychiatric and substance use disorders as risk factors for low birth weight and preterm delivery. Am Coll Obstet Gyn 100:297-304

16. Kelly RH, Russo J, Katon W (2001) Somatic complaints among pregnant women cared for in obstetrics: normal pregnancy or depressive and anxiety symptom amplification revisited? Gen Hosp Psychiatry 23:107-113

17. Louik C, Lin AE, Werler MM et al. (2007) First-Trimester use of selective serotoninreuptake inhibitors and the risk of birth defects. NEJM 356:2675-2683

18. Manber R, Blasey C, Allen JJB (2008) Depression symptoms during pregnancy. Arch Womens Ment Health 11:43-48

19. Miller LJ (1994) Use of elextroconvulsive therapy during pregnancy. Hosp Community Psychiatry 45:444-450 
20. Murray D, Cox JL (1990) Screening for depression during pregnancy with the Edinburgh Depression Scale (EPDS). J Reprod and Infant Psychol 8:99-107

21. National Institute for Clinical Excellence (NICE). Antenatal and postnatal mental health. Clinical management and service guidance Quick reference guide 45. Feb 2007 (reissued April 2007) http://www.nice.org.uk/nicemedia/pdf/CG045QuickRefGuideCorrected.pdf

22. Oren DA, Wisner KL, Spinelli M et al. (2002) An open trial of morning light therapy for treatment of antepartum depression. Am J Psychiatry 159:666-669

23. Pearson KH, Nonacs RM, Viguera AC et al. (2007) Birth outcomes following prenatal exposure to antidepressants. J Clin Psychiatry 68:1277-1278

24. Sasse J, Pilhatsch M, Forsthoff A et al. (2009) Specific aspects of treatment for women with bipolar affliction. Nervenarzt 80:263-272

25. Schäfer C, Spielmann H, Vetter K (2006) Arzneiverordnung in Schwangerschaft und Stillzeit. Urban \& Fischer, München

26. Spinelli MD, Endicott J (2003) Controlled clinical trial of interpersonal psychotherapy versus parenting education program for depressed pregnant women. Am J Psychiatry 160:555-562

27. Terman M, Terman JS, Ross DC (1998) A controlled trial of timed bright light and negative air ionization for treatment of winter depression. Arch Gen Psychiat 55:875-882

28. Tuunainen A, Kripke DF, Endo T (2004) Light therapy for non-seasonal depression. Cochrane Database of Systematic Reviews, Issue 2. Art. No.: CD004050. DOI: 10.1002/14651858.CD004050.pub2

29. Wirz-Justice A (2006) Biological rhythm disturbances in mood disorders. Int Clin Psychopharmacol 21 Suppl 1:11-15

30. Yaris F, Ulku C, Kesim M et al. (2005) Psychotropic drugs in pregnancy: a casecontrolled study. Prog Neuropsychopharmacol Biol Psychiatry 29:333-338 


\section{Abbildung 1 Edinburgh Postnatal Depression Scale (EPDS)}

Deutsche Validierung [5]

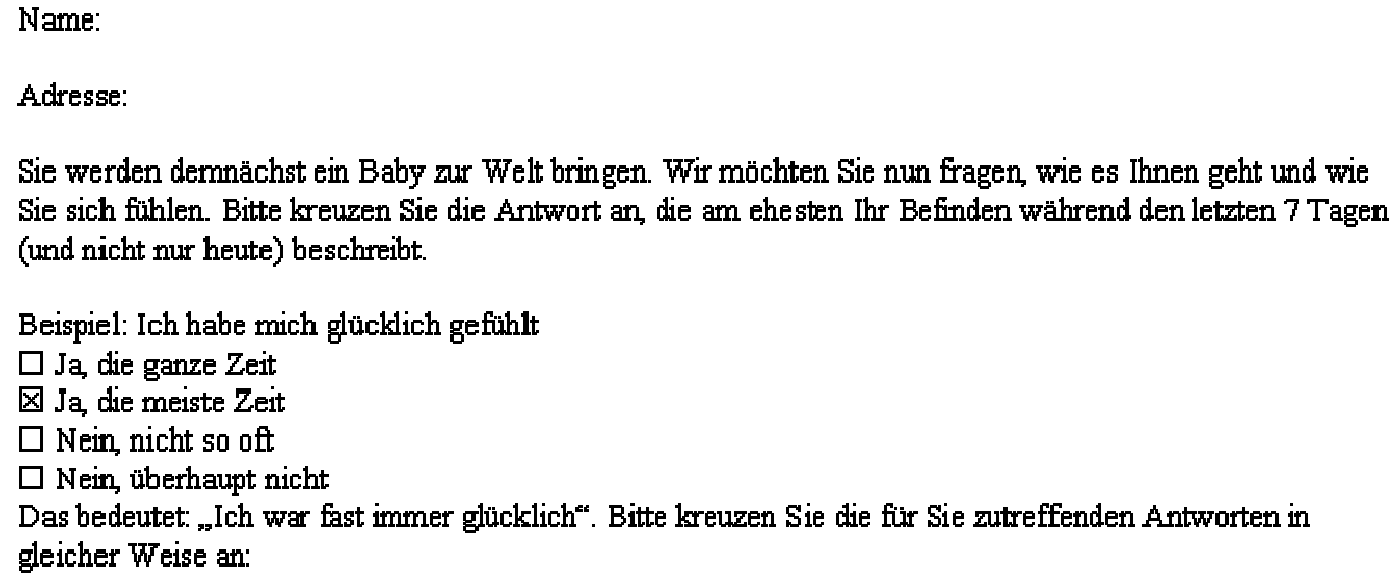

1) konnte ich lachen und das Leben von der sonnigen Seite sehen

$\square$ so wie ich es immer konnte

$\square$ nicht ganz so wie sonst immer

$\square$ deutlich weniger als früher

$\square$ überhaupt nicht

2) konnte ich mich so richtig auf etwas freuen

$\square$ so wie immer

$\square$ etwas weniger als sonst

$\square$ deutlich weniger als früher

$\square$ kaum

3) fühlte ich mich unnötigerweise schuldig, wenn etwas schief lief

$\square$ ja, meistens

$\square$ ja, manchmal

$\square$ nein, nicht so oft

$\square$ nein, niemals

4) war ich ängstlich und besorgt aus nichtigen Gründen

$\square$ nein, überhaupt nicht

$\square$ selten

$\square$ ja, manchmal

$\square$ ja, häufig

5) erschrak ich leicht bzw. reagierte panisch aus unerfindlichen Gründen

$\square$ ja, oft

$\square$ ja, manchmal

$\square$ nein, nicht oft

$\square$ nein, überhaupt nicht
6) überforderten mich verschiedene Umstände

$\square$ ja, die meiste Zeit war ich nicht in der Lage, damit fertig zu werden

$\square$ ja, manchmal konnte ich damit nicht fertig werden

$\square$ nein, die meiste Zeit konnte ich gut damit fertig werden

$\square$ nein, ich wurde so gut wie immer damit fertig

7) war ich so unglücklich, dass ich nicht schlafen konnte

$\square$ ja, die meiste Zeit

$\square$ ja, manchmal

$\square$ selten

$\square$ nein, überhaupt nicht

8) habe ich mich traurig und schlecht gefühlt

$\square$ ja, die meiste Zeit

$\square$ ja manchmal

$\square$ selten

$\square$ nein, überhaupt nicht

9) war ich so unglücklich, dass ich geweint habe

$\square$ ja, die ganze Zeit

$\square$ ja manchmal

$\square$ nur gelegentlich

$\square$ nein, niemals

10) überkam mich der Gedanke, mir selbst SIchaden zuzufügen

$\square$ ja, ziemlich oft

$\square$ manchmal

$\square$ kaum

$\square$ niemals

Die Antworten werden je nach Symptomschwere mit 0, 1, 2 und 3 bewertet. Die Fragen 3, 5, 6, 7, 8, 9, und 10 sind spiegelbildlich konstruiert (Bewertung mit 3,2,1 und 0). Die Addition der einzelnen Items ergibt den Summenscore.

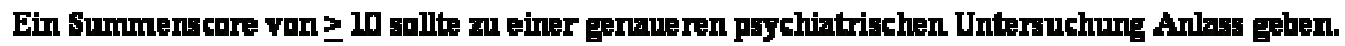


Tabelle 1: $\quad$ Folgende Punkte sollten vor dem Behandlungsentscheid zusammen mit der Schwangeren erörtert werden [21].

- Das Risiko eines Rückfalls oder einer Symptomverschlechterung in der Schwangerschaft und die Ressourcen der werdenden Mutter, mit den unbehandelten oder verschlechterten Symptomen zurechtzukommen.

- Der Schweregrad von früheren depressiven Episoden, das damalige Ansprechen auf die Behandlung, und die Behandlungspräferenzen der Betroffenen.

- Die Risiken, welche von einer abrupten Absetzung des AD bei Feststellung der Schwangerschaft ausgehen.

- Die Dringlichkeit einer Behandlung, unter Berücksichtigung der potentiellen Risiken einer unbehandelten SSD auf das Ungeborene.

- Die erhöhten Risiken durch die Einnahme eines AD in der Schwangerschaft inkl. des Risikos einer Überdosierung. 
Tabelle 2: Behandlungsmöglichkeiten in Abhängigkeit vom Schweregrad der SSD [21]

\begin{tabular}{|c|c|}
\hline $\begin{array}{l}\text { Schweregrad der } \\
\text { Depression: }\end{array}$ & Maßnahmen in der Schwangerschaft: \\
\hline Leichte Depression & 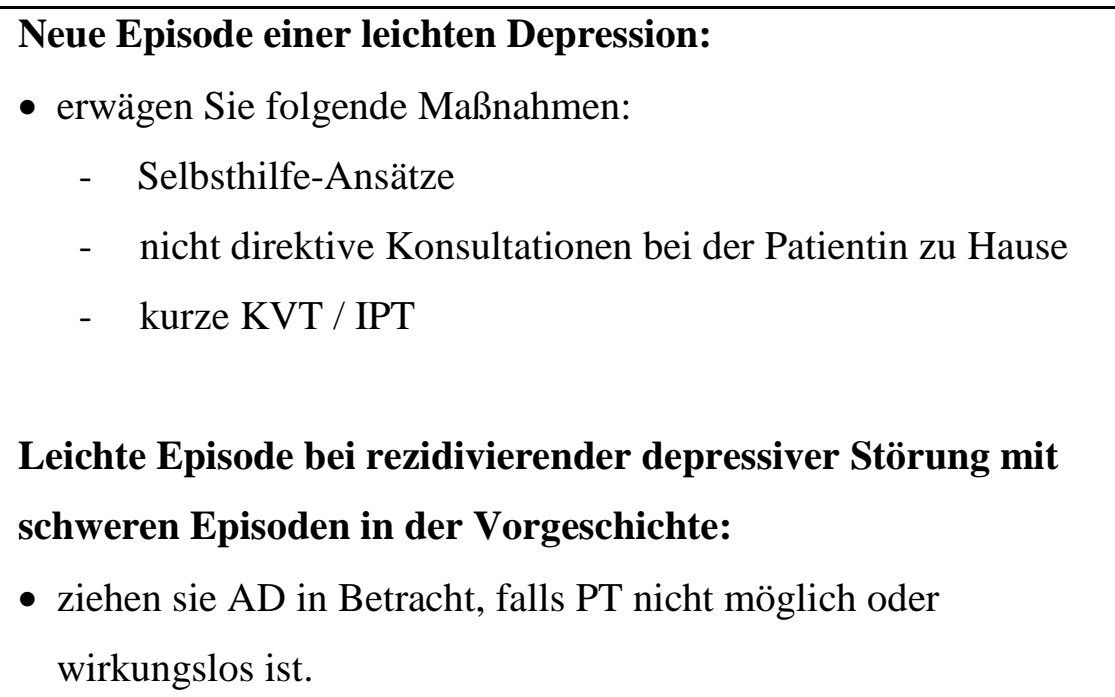 \\
\hline $\begin{array}{l}\text { Mittelgradige und } \\
\text { schwere Depression }\end{array}$ & $\begin{array}{l}\text { Neue Episode einer mittelgradigen Depression: } \\
\text { siehe leichte Depression, oben } \\
\text { Mittelgradige Episode bei rezidivierender depressiver Störung } \\
\text { oder eine schwere depressive Episode } \\
\text { - ziehen Sie folgendes in Betracht: } \\
\text { - } \quad \text { KVT / IPT } \\
\text { - AD, falls die werdende Mutter eine } \\
\text { Psychopharmakotherapie einer PT vorzieht } \\
\text { - } \quad \text { kombinierte Behandlung mit PT und AD, wenn auf PT oder } \\
\text { AD alleine nicht oder zu gering angesprochen wird }\end{array}$ \\
\hline $\begin{array}{l}\text { Therapieresistente } \\
\text { Depression }\end{array}$ & $\begin{array}{l}\text { Ziehen Sie ein AD oder eine EKT alleine vor einer Kombination } \\
\text { von mehreren Psychopharmaka in betracht. }\end{array}$ \\
\hline
\end{tabular}


Tabelle 3: Nach den NICE Guidelines ist bei antidepressiver Medikation in der SS folgendes zu berücksichtigen [21]

- Geringstes bekanntes Risiko für den Einsatz in der Schwangerschaft haben TZA wie Amitryptilin, Imipramin, Nortryptilin. Im Vergleich mit SSRIs besteht jedoch eine erhöhte Mortalität bei Überdosierung.

- Das niedrigste bekannte Risiko unter den SSRIs besteht für Fluoxetin.

- Bei Einnahme von Paroxetin im ersten Trimenon sind fötale Herzfehler beobachtet worden.

- Persistierende pulmonale Hypertension ist bei Neugeborenen aufgetreten, deren Mütter SSRIs nach der 20. Gestationswoche einnahmen.

- Venlafaxin in hohen Dosen verursacht hypertone Blutdruckwerte und hat eine im Vergleich mit anderen SSRIs und einigen TZA erhöhte Toxizität bei Überdosierung. Es kann zu stärkeren Absetzphänomenen kommen.

- Unter allen Antidepressiva kann es zu Absetzphänomenen und Intoxikationen der Neugeborenen kommen, wobei diese Phänomene in den meisten Fällen mild und selbstlimitierend verlaufen.

- Frauen, die unter Paroxetin schwanger werden sollte zum Absetzen der Medikation geraten werden. 


\section{Infobox}

Mehr Information zum Thema:

Psychopharmaka in der SS: www.frauen-und-psychiatrie.de

Lichttherapie (allgemein): www.cet.org (non-profit Organisation für PatientInnen und ÄrztInnen); Lichttherapie (Praxis) für MedizinerInnen und PsychologInnen: www.chronotherapeutics.org; Basel Lichtforschung: www.chronobiology.ch 